\title{
RFID based Women Safety System with Automatic Street Light Control System
}

\author{
Shruthi A B ${ }^{1}$, Shivanand Gowda K R $\mathbf{R}^{2}$, Ramachandra A $C^{3}$ \\ PG Student, VLSI Design \& Embedded Systems, Alpha College of Engg, Bengaluru, India ${ }^{1}$ \\ Assistant Professor, Dept of Electronics \& Communication Engg, Alpha College of Engg, Bengaluru, India ${ }^{2}$ \\ Professor \& HOD, Dept of Electronics \& Communication Engg, Alpha College of Engg, Bengaluru, India ${ }^{3}$
}

\begin{abstract}
Safer transportation of women employees in the night or even in the day time has been a critical issue as it is often found that if they miss the bus/cab or ride the wrong bus/cab with no way to track them. This project intends to find yet another solution to solve this problem by developing a safety system that will control the entry and exit of employees from the buses through an efficient methodology. The proposed system will control the entry and exit of employees using RFID (Radio Frequency Identification) and GSM technologies to ensure the entering and exiting of all employees in a safer manner. The system will do all the process and allow the employee to be tracked while entering and leaving the bus. If the bus journey is successful from the source to destination, it will send an SMS to the management to inform its departure and arrival. It also gives the continuous information about the bus with the help of GPS. If the driver exceeds the speed limit, a message will be sent to the management that the driver is rash driving. In case of accidents, for the safety of employees, it also has Accident Detection System to inform the Ambulance and Police of the accident site and arrange for necessary steps to control the situation. The employee can be tracked by the management and the guardian throughout the travel with the help of this system. It also has automatic street light control system that can be implemented in low density areas. It helps to automatically turn ON and OFF according to the intensity of light and the movement of any vehicles.
\end{abstract}

Keywords: Women Safety System, accident detection, speed limit alert, automatic messaging system, automatic streetlights.

\section{INTRODUCTION}

In the recent years, Indian women are more independent. For working women, travelling to office and back to home is a daily and necessary need. The safety of women during this travel is very important and it is obviously the responsibility of the office management. There is a need to ensure the boarding and de-boarding of each and every employee on both sides of the travel. Also, it is important to know the location of the office transport and the employees throughout the travel. The proposed safety system is designed to control the entering/exiting of employees from the bus. This system does several tasks, including identifying personal information (eg. Name) of each employee using RFID tag, which will exchange the data with the RFID reader via radio waves and displaying each employee name into LCD display. This will let the driver to know the number of employees inside the bus and the employees who departed from the bus. In addition, if the bus depart and arrive successful from the source to destination, it will inform the management through an SMS about its successful departure and arrival. It will also provide continuous information about the bus to the management and a preset number of employee's guardian with the help of GPS. Though not within strictly in the scope, the same data can be used to assess the time of departure and arrival, number of employees travels each day.

The system also detects the accidents and sends message to the preset numbers with the use of GSM and GPS technologies. GPS is used for taking the coordinates of the site of the accident while GSM is used for sending the coordinates to cell phones. To make this process all the controls are made using Arduino. The components used are Vibration Sensor, which detects the accident and in turn sends the signals to Arduino. At this point the Arduino takes control and starts collecting the coordinates received from the GPS which are later sent to the emergency contact numbers like Police, Ambulance and to the office management by using the GSM Module. The system also detects the speed of the vehicle using an analog system. If the driver exceeds the speed limit set by the management, then the system sends an SMS to the management informing that the driver is rash driving. The Women Safety System can be fitted in the office buses and can be tracked by the management with the help of software. In low density areas, usually no streetlights are present to save the power and for cost reduction. So, in this project, we also have automatic streetlights which turns on only when there is dark and if any vehicles or human beings move in the road. It helps in saving the power and the cost efficiently. Also, helps in giving the security to the people who travel or walk during the night in low density areas. 
Vol. 6, Issue 6, June 2017

\section{HARDWARE \& SOFTWARE REQUIREMENTS}

\subsection{HARDWARE REQUIREMENTS}

Arduino Mega 2560 is used to control the unit. It is a microcontroller based on ATMEGA2560. ATMEGA2560 is a low power CMOS 8-bit microcontroller based on RISC architecture. It helps to achieve high performance using low power consumption. An RFID reader gathers information from the RFID tag which helps to track the objects. The data is transferred using radio waves. RFID reader has an antenna which sends power as well as data and commands to the tags. It is like an access point for RFID tagged items so that the data in the tag is made available to the applications. RFID tags are made from an integrated circuit (IC) that is connected to antenna for transmitting and receiving the signals. The tag chip has a memory to store the product's EPC (Electronic Product Code) and other information that can be read and tracked by RFID readers. The RFID tags must be in the range of RFID reader i.e. 3 to $300 \mathrm{ft}$ to communicate with each other. In this project, RFID Reader and tags are used for identification of the employees during boarding and de-boarding of the bus. The Global Positioning System (GPS) is a global navigation satellite system (GNSS) which provides geographical location and information about time to a GPS Receiver. It works in all weather conditions, anywhere on or near the earth. But, there should be a clear line of sight (without any obstacle) to minimum of four GPS satellites. GPS satellite sends signal to the GPS receiver along with the exact time of the signals sent. With this, the GPS can give the information that how far it is from the satellite by subtracting the time the signal was transmitted from the time the signal was received. The GPS receiver will also have the information about the exact position of the satellites in the sky at the time they sent the signal. Hence with the travel time of the GPS signals from three satellites and their exact position in the sky, the GPS receiver calculates the position in three dimensions - east, north and altitude. In the project, GPS is used to get the location of the bus and the employee whenever needed. It gives the location information of the bus and the employee continuously which helps to monitor. GSM is an acronym that stands for Global System for Mobile Communications which is an international standard for mobile telephones. It is a second generation network and hence it is often referred to as 2G. GSM mainly supports incoming and outgoing voice calls, SMS (Simple Message System) services, and data communication. GSM digitizes and compresses data to send it through a channel along with the other streams of user data. It operates at a frequency range of 900 or $1800 \mathrm{MHz}$. GSM is used in the project for sending and receiving SMS. It is used to send SMS during boarding and de-boarding of the employees, in case of accidents and in case of rash driving. It is also used to receive the SMS sent by the guardians to know the location of the employees and to send back an SMS with the location information.

A piezoelectric sensor is used as vibration sensor to detect the accidents. A piezoelectric sensor is a device measures the changes in pressure, acceleration, temperature, strain or force by converting them to an electrical charge by using piezoelectric effect. When there is an accident, piezoelectric sensor detects the vibrations. If the vibration exceeds the specified threshold limit, a message will be sent informing about the accident and its location using GPS. A liquidcrystal display (LCD) is a flat panel display, electronic visual display, or video display that uses the light modulating properties of liquid crystals. Liquid crystals do not emit light directly. Here, we use a monochromatic 20x4 alphanumeric LCD. 20x4 means that 20 characters can be displayed in each of the 4 rows of the 20x4 LCD and hence a total of 80 characters can be displayed. LCD Display is used in this project as a part of Women Safety System and is used to display some necessary information in front of the driver like employee boarded and de-boarded, GPS location etc. A DC Motor converts DC electrical energy to mechanical energy and also converts mechanical energy to dc electrical energy. Here, a dc motor is used to present the speed limit module. The DC motor is connected to Arduino with the help of four 10K resistors. Mechanical energy is applied on the DC motor run it and the output is noted. Practically, this DC motor represents the wheels of the vehicle which is connected to the speedometer to track the speed of the moving vehicle. A light dependent resistor or a photo-resistor is a variable resistor controlled by the light. It is a light sensitive device. As the intensity of the light increases, the resistance of LDR decreases hence the conductivity $\mathrm{f}$ the device increases. This phenomenon is known as Photoconductivity.

LDR is made up of a semiconductor material with high resistance. In the dark, the resistance of LDR is very high whereas in the light, the resistance is low. When there is a light incident on the device, the electrons in the semiconductor material jump from valence band to conduction band. When there is more light incident on it, more electrons jump to conduction band and hence there is more current flow in the device when the circuit is closed and the resistance decreases. Here, LDR is used to control the streetlights which turns on or off according to the incidence of light on it. IR Transmitter \& Receiver module works on the principle of reflection of infrared rays from the incident surface. IR transmitter is an IR LED which emits IR rays continuously. When an obstacle comes in between the transmitter and receiver, these IR rays are reflected back and captured. When there is no obstacle, there rays are absorbed by the IR Receiver and the IR rays remain uncaptured. Here, IR Transmitter \& Receiver module is used to control the streetlights which turns on or off according to the presence of vehicle. SMD stands for Surface Mount Device. SMD LED module can be mounted on printed circuit boards using Surface Mount Technology (SMT). These are widely used in LED lamps, automobile interior lighting, home illumination etc. Here, we are using LED lights to 
represent street lights. To control a block of streetlights, we need to have a circuit which includes a relay module, 7812 voltage regulator and a battery. The circuit is as shown in the fig 1.

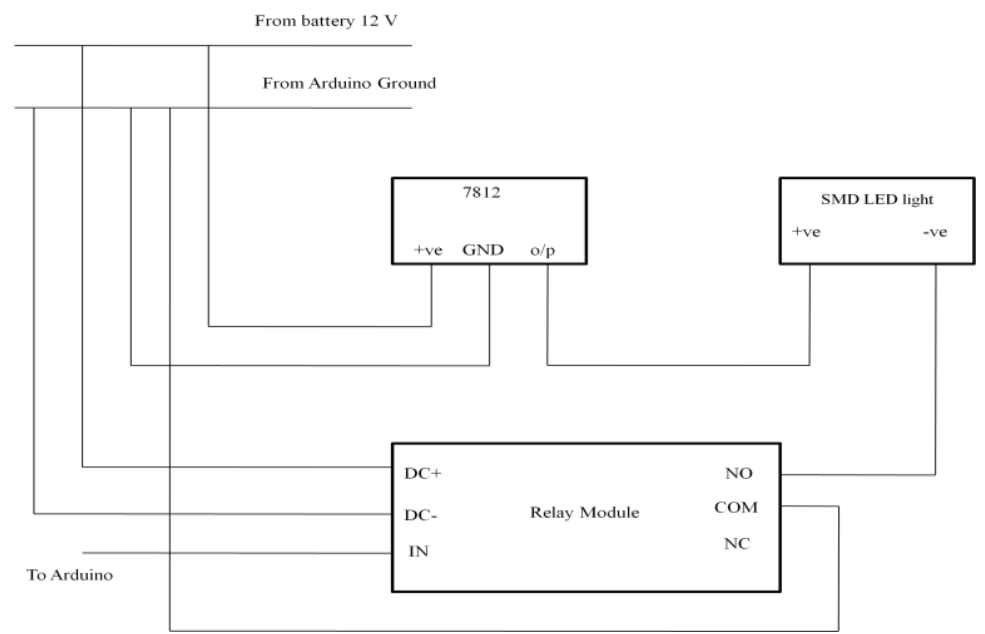

Fig 1. Control Circuit for Street lights

\subsection{SOFTWARE REQUIREMENTS}

The Arduino IDE (Integrated Development Environment) has a text editor for writing the code, a message area to display the errors and warnings, a text console, a tool bar with buttons and few menu options. Arduino IDE helps us to connect to the Arduino hardware so that we can upload the programs. The program is developed using embedded C. C is the most widely used programming language for embedded processors/controllers. AT Commands are used to control GSM modems to communicate with them and do the required functions. The set of commands consists of a series of short text strings. Commands are used for operations such as dialling, hanging up and sending messages. In the beginning, a set of commands are required to establish a connection between the mobile operator and the GSM modem. In this project, we are calling the AT commands from the program for sending, receiving and deleting messages. All these operations will be carried out automatically when the program runs without any manual intervention for sending AT commands.

\section{PROPOSED SYSTEM}

\subsection{Block Diagram}

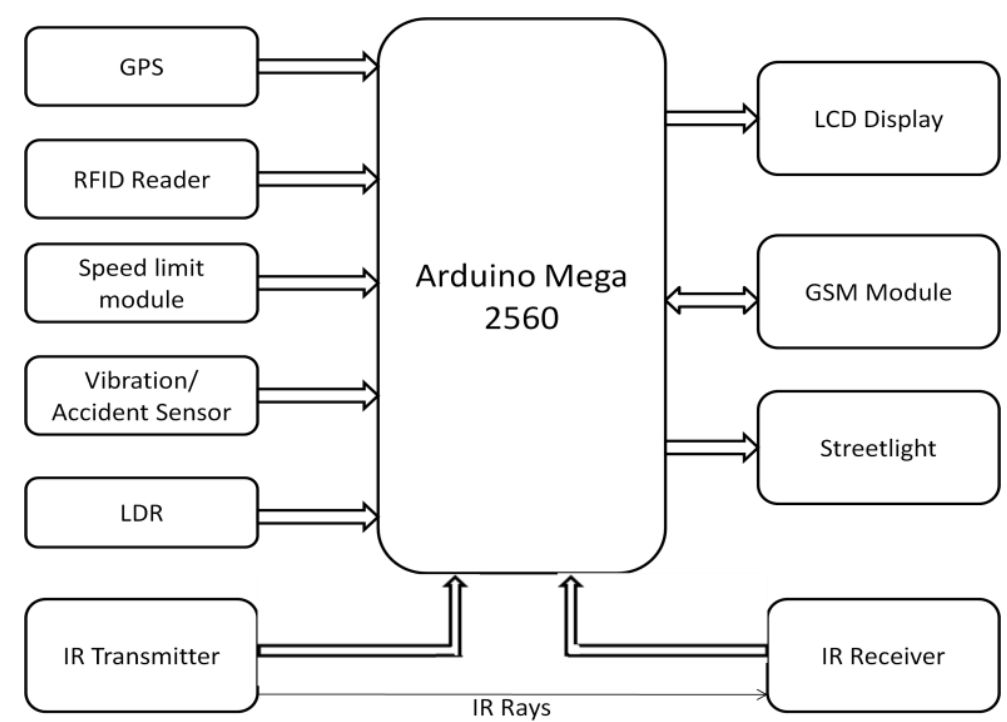

Fig 2. Block Diagram of proposed system

\subsection{Working Principle}

When an employee boards the bus, the employee swipes her identity card which has RFID tag in it. The RFID tag will be mapped to the individual with her personal details like name, address, mobile number and an emergency contact 
number. Tracking the employee details is done with the help of RFID tags and RFID Reader. As employee boards the bus and swipes the card, employee's name and her location details will be displayed on the LCD Display and the same details will be sent via SMS to the office management and to any of the registered number of the guardian that the employee has boarded the bus. This will also let the driver know the number of employees inside the bus and the employees who departed from the bus. The location details of the employee is tracked with the help of GPS Receiver and GPS Antenna. During the travel, if the driver exceeds the speed limit, an SMS is sent to the office management that the driver is rash driving. Here, the DC motor used in the project represents the wheel of the bus. The DC motor is connected to Arduino to sense the speed of the bus when we rotate DC motor or apply the mechanical energy. The analog reading of the speed is read continuously by the Arduino. A threshold speed is set by the management in the program. Arduino displays the speed reading when the bus exceeds the speed threshold set by the management. Immediately an SMS will be sent to the management saying that the driver is rash driving. If the bus meets with any accident, immediately, an SMS with the accident location details will be sent to office management, emergency contact numbers for help and to the boarded employees' registered numbers. In case, if any employee has de-boarded the bus before the accident, then those employees' guardians will not get the alert message about the accident. Emergency Contact numbers for help during accident include the contact numbers of police, ambulance and emergency contact numbers of the office and the employees. The accident will be detected with the help of piezoelectric sensor which detects the vibration. During the journey, if any of the employee's guardian wants to know the location of the bus or the employee, the guardian can send an SMS with the keyword "<employee name> location" to the GSM module. The program recognizes the keyword and sends location details to the employee's guardian number. If the message is sent by any of the numbers other than the employee guardian's registered number, the location details will be sent only to the registered number of that particular employee. This feature is implemented to maintain the privacy of the employee location details during the travel. The received message is deleted immediately after sending the location details. The system is configured such that it responds to only the messages with the specified keyword. If any other messages, it checks for the string and immediately deletes those messages. When the employee reaches the destination, while getting down from the bus, employee swipes the card again. At this time also, an SMS will be sent to the office management and to the registered number of the guardian that the employee has reached the destination. Therefore, basically the system helps to track the employees and the bus continuously and efficiently throughout the travel. In low density areas, we can implement automatic streetlights which automatically turns on and off only if necessary. During day time, when there is sunlight, the lights will be turned off. This is because of the LDR (Light Dependent Resistor) we have used in the circuit. During night, normally, the lights will be turned off. Only when there is movement of any object (vehicles or human beings), the lights get turned on automatically. Soon after the object moves away from the place, the lights get turned off automatically. IR Transmitter and Receiver helps to detect the objects. The transmitter continuously emits IR rays to the IR receiver. When an object comes in between the transmitter and receiver, the IR rays gets reflected back to the transmitter and hence the obstacle is detected. When there is no object in between the transmitter and receiver, the receiver absorbs the IR rays that are emitted from the receiver. Hence, the automatic streetlights provides safety as well as saves power.

\section{RESULTS}

The implementation and model of the proposed system is as shown in the fig 3 below. It has GPS, GSM unit, RFID unit, Speed limit module with DC motor and Automatic Streetlights controlled by IR Transmitter \& Receiver module, LDR circuit and a control unit which has relays, 7812 voltage regulator and a battery.

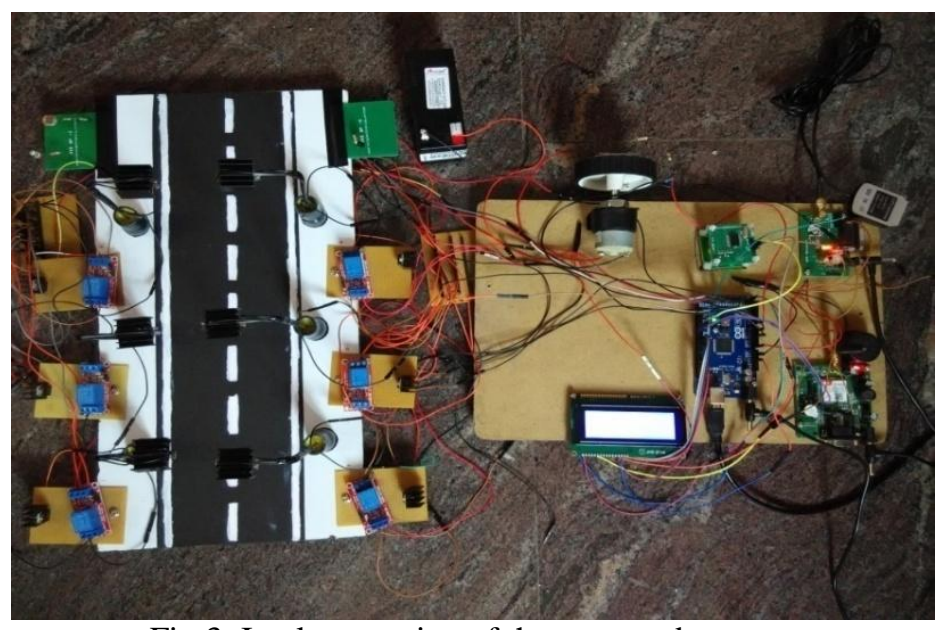

Fig 3: Implementation of the proposed system 
All these are controlled by a single Arduino. During the day time, when there is sunlight, obviously the lights will be switched off. Also, during the night time, when there are no movements of vehicles or human beings, the lights will be switched off by default. During the night time, by default, the lights will be switched off. When there is a vehicle or human beings approaching towards the block of lights, the IR transmitter \& Receiver module detects them and the lights will get turned on automatically.

4.2 Messages received in all the features
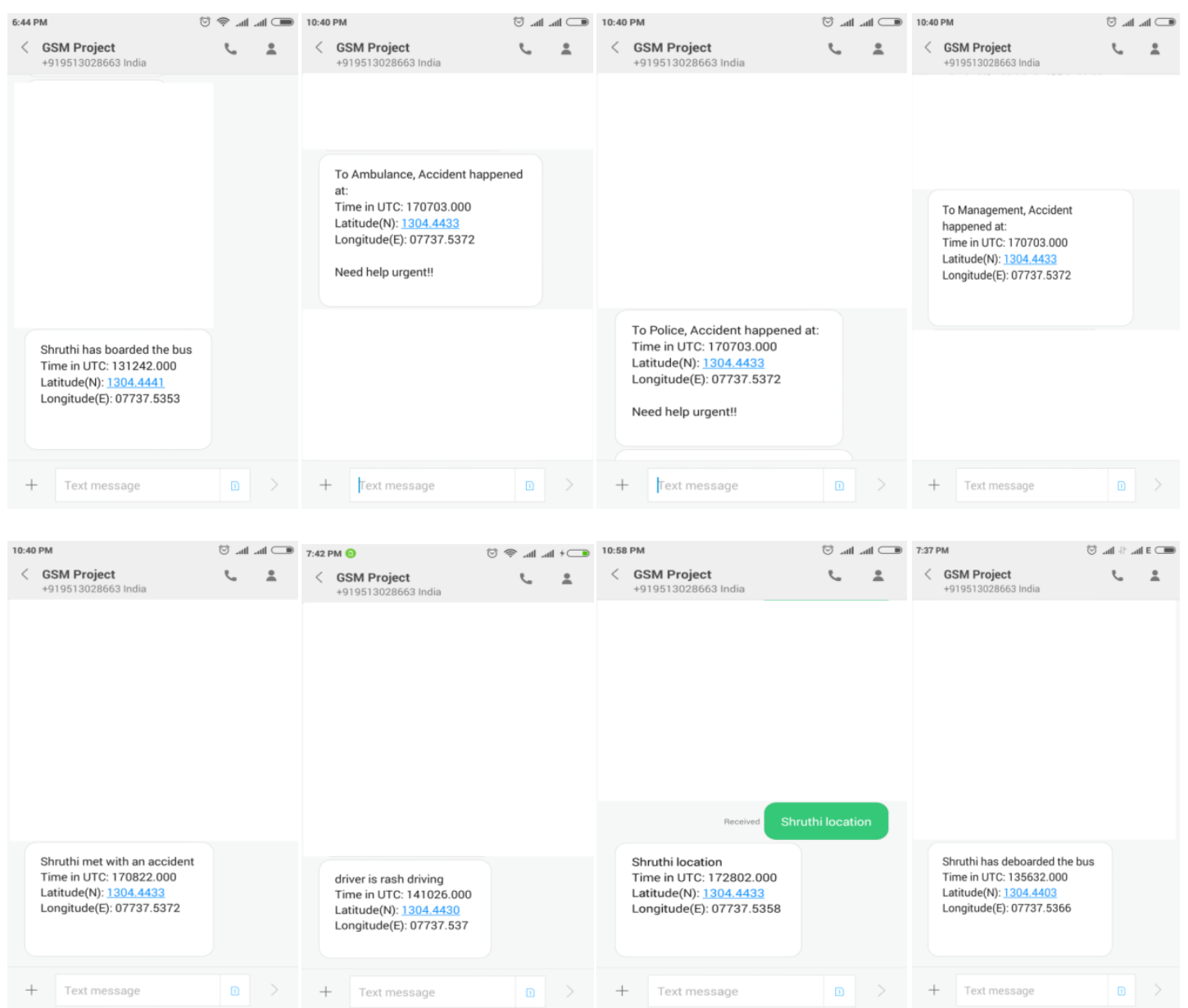

Fig 4: When employee boarded, information about accident to Ambulance, Police, Management and Employee guardian, when exceeded speed, location request and when employee is deboarded respectively

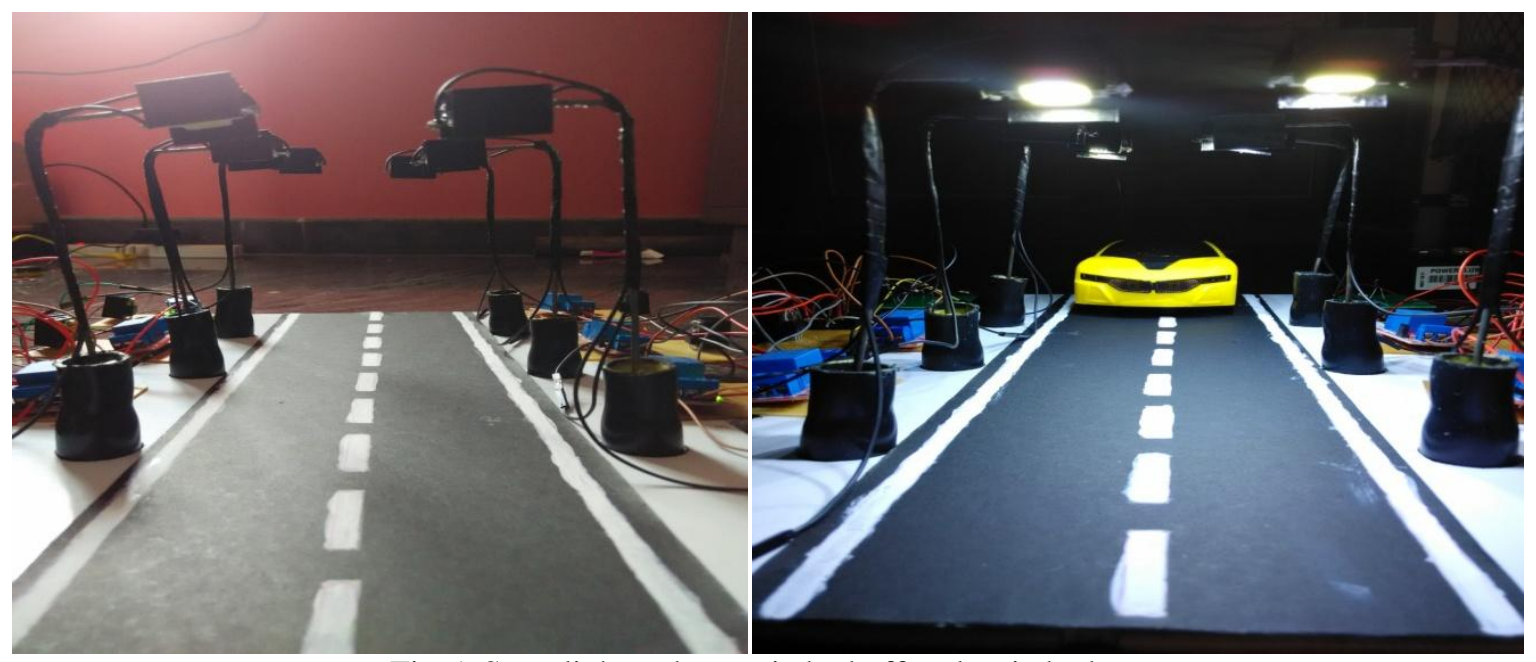

Fig 5: Streetlights when switched off and switched on 
Vol. 6, Issue 6, June 2017

\section{BENEFITS, LIMITATIONS \& APPLICATIONS}

5.1 Benefits

- The RFID based attendance system is advantageous over the conventional method of IN and OUT monitoring.

- This will help in automatic storage of attendance and the attendance is very much accurate.

- The system allows tracking the employees easily and efficiently in case of emergencies.

- Immediate medication can be provided to the accident victims in the remote areas.

- The system provides good safety for women employees travelling in the late evening as they can be tracked by the management and the guardians continuously throughout the travel.

- Using Arduino to control the unit, it ensures fast operation, cost effectiveness and low power consumption.

- The design is simple and reliable.

- Automatic streetlights provides safety as well as saves power by turning off the light when unnecessary.

5.2 Limitations

- In some places where there is no provision of GSM networks it is difficult for communication.

5.3 Applications

- The safety system can be applied in school buses for the safety of children so that the children will be tracked by the parents. In case of accidents, the parents will be notified and appropriate actions can be taken.

- The system can be used in the rental vehicle services which helps to track them easily.

- The system can be used for stolen vehicle recovery. Both consumer and commercial vehicles can be outfitted with RF or GPS units to allow police to do tracking and recovery.

- It can be used in transit tracking which is a temporary tracking of assets or cargoes from one point to another. Users will ensure that the assets do not stop on route or do a U-Turn in order to ensure the security of the assets.

\section{CONCLUSION AND FUTURE WORK}

\subsection{Conclusion}

This system has many features like Employee tracking system, accident detection and messaging system and Speed limit module for sensing exceed in speed limit are combined together for the safety of employees. The management and the guardian both are alerted in case of emergencies so that necessary actions can be taken immediately. Employee can be tracked by both the management and the guardian continuously throughout the travel. It also has automatic street light control system which saves the energy when there is no requirement of light.

\subsection{Future Work}

- Informing by SMS can be bettered by developing an application for the users i.e the guardians and the management where the updates will be sent via notifications.

- The system can be enhanced by including an automatic brake locking system. Usually, during accidents, the drivers lose control over the brakes and fail to stop the vehicle. When the vibration sensors get triggered during accidents, Arduino should be linked to the devices which can lock the brakes and helps to weaken the impact of the accident.

\section{REFERENCES}

[1] Nitin Shyam, Narendra Kumar, Maya Shashi, Devesh Kumar, "SMS Based Kids Tracking and Safety System by Using RFID and GSM", International Journal of Innovative Science, Engineering \& Technology, Vol. 2 Issue 5, May 2015.

[2] A.Gowthaman, R.Mohanraj, A.Anadhan, S.Mohan, " Implementation of school children tracking system and transportation safety enhancement by using RFID technique", International Journal of Emerging Technology in Computer Science \& Electronics (IJETCSE) ISSN: 0976-1353 Volume 21 Issue 2 - APRIL 2016.

[3] Maryam Said Al-Ismaili, Ali Al-Mahruqi, Dr. Jayavrinda Vrindavanam, " Bus Safety System for School Children Using RFID and SIM900 GSM MODEM", International Journal of Latest Trends in Engineering and Technology (IJLTET), Vol. 5 Issue 1 January 2015.

[4] C.Prabha, R.Sunitha, R.Anitha, "Automatic Vehicle Accident Detection and Messaging System Using GSM and GPS Modem", International Journal of Advanced Research in Electrical,Electronics and Instrumentation Engineering, Vol. 3, Issue 7, July 2014.

[5] Mr.Dinesh Kumar HSDK, Shreya Gupta,Sumeet Kumar,Sonali Srivastava, "Accident Detection and Reporting System Using GPS and GSM Module", Journal of Emerging Technologies and Innovative Research (JETIR), Volume 2, Issue 5, May 2015.

[6] D.Narendar Singh,Ravi teja ch.v.,"Vehicle Speed Limit Alerting and Crash Detection System at Various Zones",International Journal of Latest Trends in Engineering and Technology (IJLTET),Vol. 2 Issue 1 January 2013.

[7] Ankita Mishra, Jyoti Solanki, Harshala Bakshi, Priyanka Saxena,Pranav Paranjpe: "Design of RF based speed control system for Vehicles" International Journal of Advanced Research in Computer and Communication Engineering Vol. 1, Issue 8, October 2012.

[8] Sindhu.A.M,Jerin George,Sumit Roy,Chandra J,"Smart Streetlight Using IR Sensors",IOSR Journal of Mobile Computing \& Application, Volume 3, Issue 2.,Mar. - Apr. 2016.

[9] Deepak Kumar Rath,"Arduino Based: Smart Light Control System",International Journal of Engineering Research and General Science Volume 4, Issue 2, March- April, 2016. 